\title{
Editorial
}

\section{Nanomaterials for Solar Energy Harvesting and Storage}

\author{
Peng Hu, ${ }^{1}$ Yuebin Cao, ${ }^{2}$ Ning Han, ${ }^{3}$ Danping Wang, ${ }^{4}$ and Jiangchao Chen ${ }^{5}$ \\ ${ }^{1}$ Chinese Academy of Sciences, Beijing 100190, China \\ ${ }^{2}$ Hanyang University, Seoul 04763, Republic of Korea \\ ${ }^{3}$ City University of Hong Kong, Kowloon, Hong Kong \\ ${ }^{4}$ Energy Research Institute, NTU (ERIN), Singapore 637141 \\ ${ }^{5}$ University of South Dakota, Vermillion, SD 57069, USA \\ Correspondence should be addressed to Peng Hu; pengh@home.ipe.ac.cn
}

Received 10 September 2015; Accepted 13 September 2015

Copyright (c) 2015 Peng Hu et al. This is an open access article distributed under the Creative Commons Attribution License, which permits unrestricted use, distribution, and reproduction in any medium, provided the original work is properly cited.

Solar energy harvesting and storage have nowadays attracted tremendous research efforts due to the increasing energy challenges that fossil fuels are being rapidly consumed by modern technology. For material chemists, how to develop materials that are effective in harnessing solar energy is the primary step to tackle this challenge. Apart from selection of materials with intrinsic physical properties to utilize solar energy, scientists have also been studying the unique properties when the particle size reduced to the nanometer scale. Nanomaterials, which have high surface-to-volume ratio, cover a variety of shapes of nanoparticles, nanorods, nanoporous framework, and so on. One can also easily tune the optical and charge transfer properties by changing the size of semiconductor nanomaterials. The chemical properties such as catalytic activity can also be remarkably changed with increased surface atoms of nanocatalysts.

This special issue is mainly dedicated to the synthesis of functional nanomaterials which can be used to harvest or store solar energy. As materials innovation has great potential to advance this area, most of the articles in this special issue describe the latest preparation methods and some of the current progress of semiconductor-based materials.

The desire to make solar energy into usable energy format has led to the fast development of solar cells, a direct way to convert solar energy into electricity. The ideal candidate materials to be used in solar cells should possess proper band gap to harvest as much sunlight as possible, good charge transport properties, excellent stability, and cheap cost. The preparation of $\mathrm{CuInS}_{2}$ thin films has been reported in two papers. R.-F. Guan and coworkers prepared the $\mathrm{CuInS}_{2}$ thin films via one-step electrodeposition method. They investigated the effects of synthesis parameters on the structure, morphology, and optical and electrical properties on the final $\mathrm{CuInS}_{2}$ thin films, and optimal preparation conditions were proposed. In another paper, R.-F. Guan and coworkers used magnetron sputtering followed by sulfurization method to prepare $\mathrm{CuInS}_{2}$ thin films. Optimized sputtering power, sputtering gas pressure, heat treatment temperature, and duration were reported. With detailed study on the synthesis parameters in each paper, these two papers will certainly help peer researchers for the facile preparation of $\mathrm{CuInS}_{2}$ based solar cells.

As a special type of solar cell, Dye-Sensitized Solar Cells (DSSCs) use photoexcited electrons generated from the conduction band of $\mathrm{TiO}_{2}$ thin films to generate electricity with the assistance of dye molecules. The key factors to prepare an effective DSSC are high surface area of $\mathrm{TiO}_{2}$ thin film, efficient charge injection from dye molecules to $\mathrm{TiO}_{2}$, and minimal loss of electrons from $\mathrm{TiO}_{2}$ to outer circuit. Kim and coworkers developed $\mathrm{TiO}_{2}$ nanobranch/nanoparticle hybrid structure to increase the surface area of thin film and thus the loading of dye molecules. Light harvesting efficiency is greatly promoted, leading to tripled incident photon conversion efficiency (IPCE) compared with bare nanobranch thin films.

A comprehensive review on the hybrid organic-inorganic perovskites solar cells is given by the $\mathrm{Xu}$ group, covering perovskite synthesis methods, hole transport materials, photoanode morphologies, and outlook and challenges for perovskites based solar cells. 
Another alternative and interesting approach to utilize solar energy is through the processes of photocatalysis or artificial photosynthesis to convert solar energy into chemical energy to drive certain chemical reactions, for example, water splitting or photodegradation of organic pollutants. P. Dong and coworkers synthesized $\mathrm{Ag}_{3} \mathrm{PO}_{4}$ triangular prism by a simple coprecipitation method which was used as an excellent photocatalyst for organic compounds degradation under light irradiation.

Biomass, the nature's way of storing solar energy, is also covered in our special issue. Y. Zhang and coworkers used corn straw as the raw material, ionic liquids as solvents, and acids as catalysts to study the effects of hydrolysis conditions on reducing sugar concentration, and optimal hydrolysis parameters were provided.

White light emitting diodes (LEDs) have been acknowledged as highly efficient and green light sources. When coupled with solar cells, the white LEDs can provide efficient lighting without consuming other energy resources. R.-F. Guan and coworkers synthesized $\mathrm{Y}_{2.94-x} \mathrm{Al}_{5} \mathrm{O}_{12}(\mathrm{YAG}): \mathrm{Ce}_{0.06} \mathrm{Pr}_{x}$ phosphors with various $\mathrm{Pr}^{3+}$ concentrations by coprecipitation method. They investigated the influence of $\mathrm{Pr}^{3+}$ doping concentrations on the phases, luminescent properties, and energy transfer phenomenon from $\mathrm{Ce}^{3+}$ to $\operatorname{Pr}^{3+}$.

Peng $\mathrm{Hu}$ Yuebin Cao Ning Han

Danping Wang Jiangchao Chen 

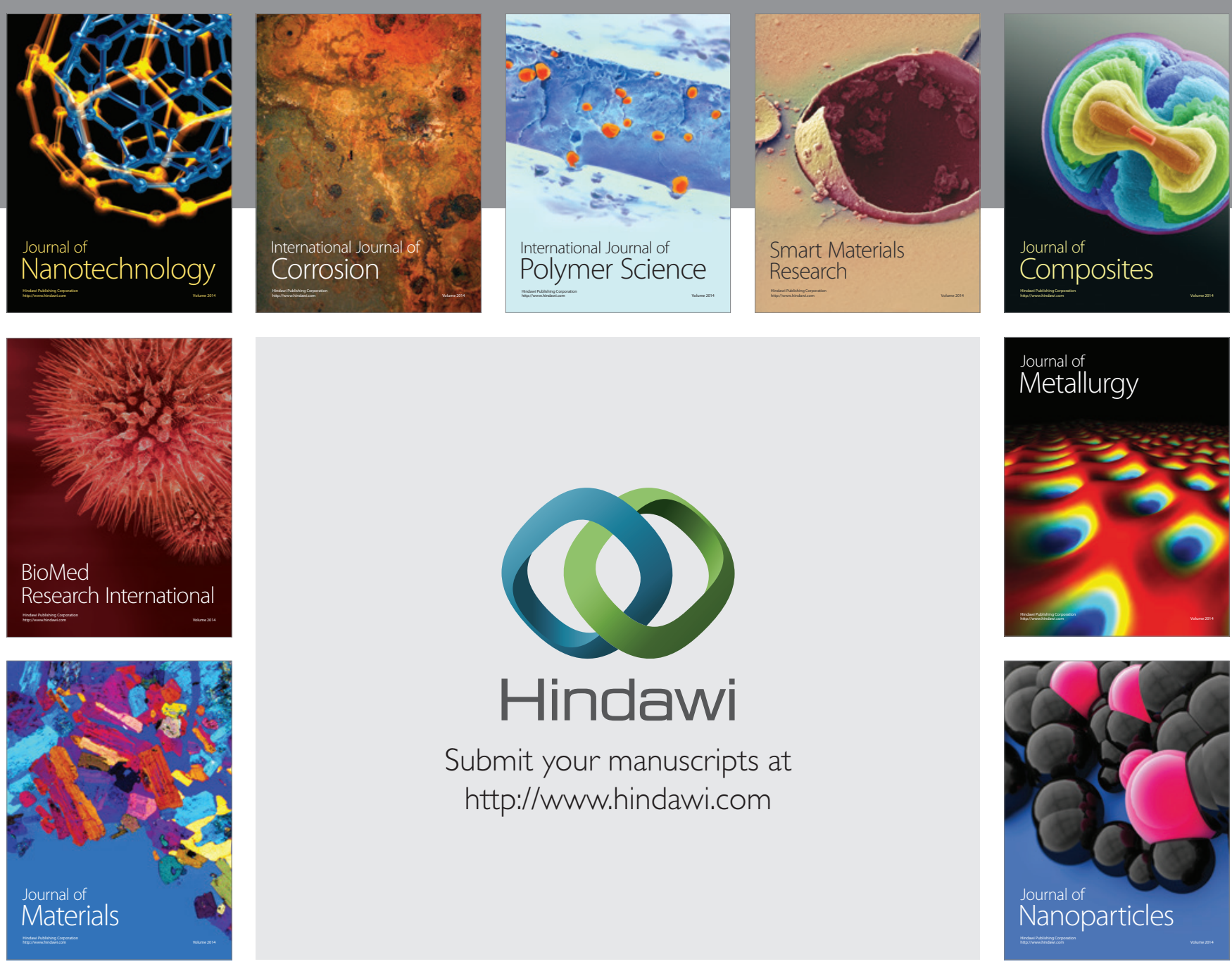

Submit your manuscripts at http://www.hindawi.com
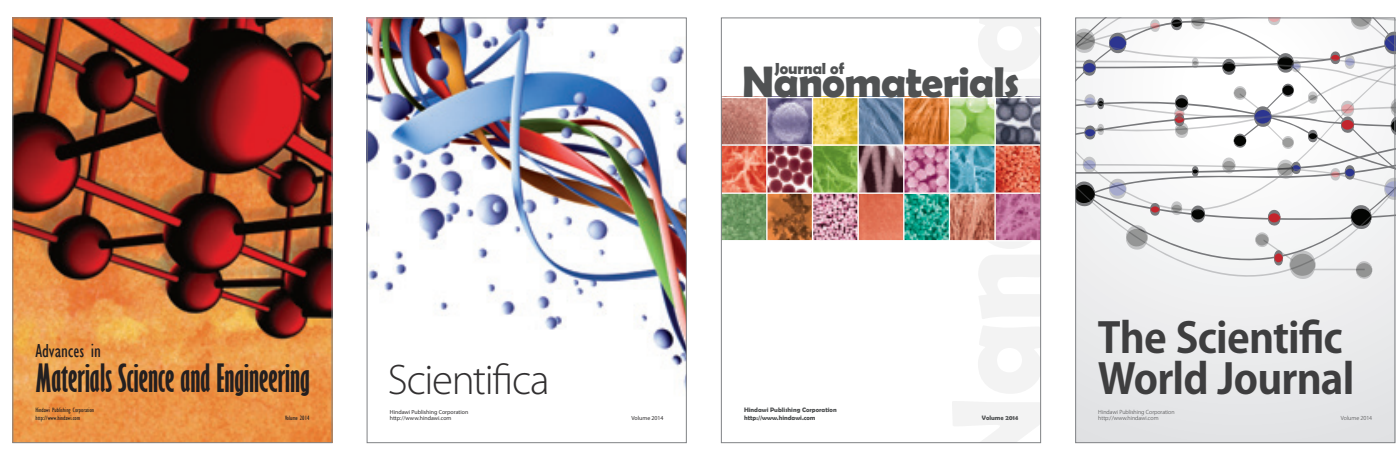

\section{The Scientific World Journal}
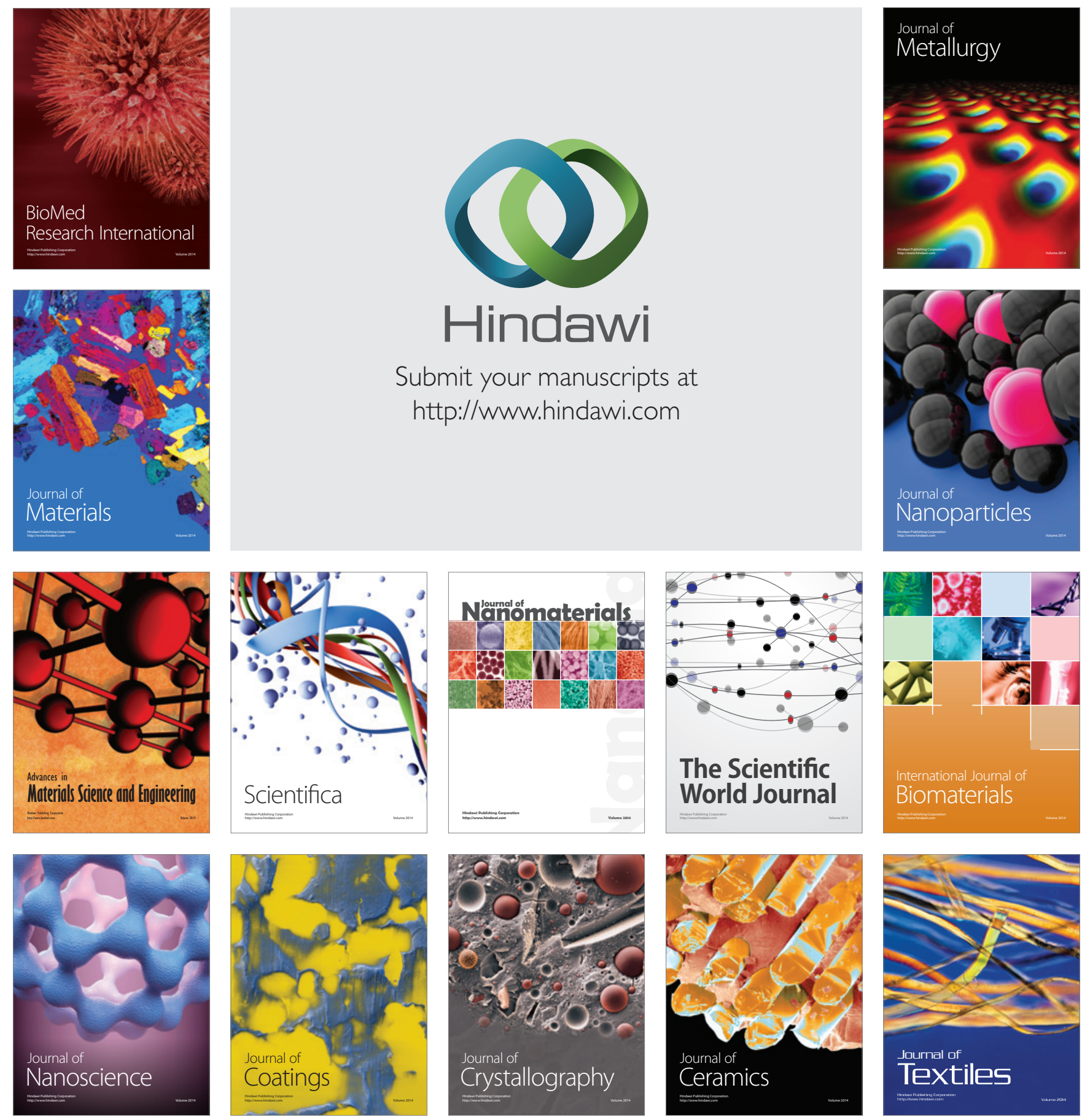